\title{
HISTORIA DE LA IDEA ANTROPOBIOLÓGICA
}

\author{
Leopoldo José Prieto López \\ Universidad Francisco de Vitoria. Madrid
}

Resumen: El artículo tiene dos partes. En la primera se analiza la idea antropobiológica en sí misma, considerando sus aspectos e implicaciones fundamentales. En la segunda parte, en cambio, desde una perspectiva histórica, se exponen algunos de los desarrollos más representativos de la idea antropobiológica, como ocurre en la obra de Aristóteles, Tomás de Aquino, Kant, Herder, von Uexküll y Lorenz.

Palabras clave: Rasgos biológicos humanos atípicos, inespecialización morfológica, indeterminación de la conducta, nueva valoración de la corporalidad humana.

\section{History of the anthropobiological idea.}

Abstract: This paper has two parts. In the first one the anthropobiological idea is analyzed in itself, regarding its fundamental aspects and implications. In the second part, instead, is adopted a historical perspective, that consists in exposing some of the most representative developments of the anthropobiological idea, as occurs in the works of Aristotle, Thomas Aquinas, Kant, Herder, von Uexküll and Lorenz.

Key words: Atypical human biological features, morphological inespecialization, indetermination of behaviour, reappraisal of human corporality.

Recibido: 14/10/2017 Aprobado: 5/11/2017 
En este trabajo se recogen las ideas fundamentales expuestas en la ponencia titulada Historia de la idea antropobiológica, presentada en el Seminario "Humanos e inhumanos: Qué nos une a y qué nos separa de las restantes especies animales" (Ávila, 13-14 de julio de 2017). En él voy a exponer dos cuestiones estrechamente relacionadas entre sí. En primer lugar voy a tratar de la idea antropobiológica en sí misma. En segundo lugar vamos a ver, con la necesaria brevedad, algunos desarrollos históricos, tanto antiguos como modernos, de dicha idea.

En lo que se refiere a la idea antropobiológica en sí misma pretendo poner de manifiesto que, en mi opinión, dicha idea parece un modelo adecuado para la investigación de la complejísima naturaleza humana. La idea antropobiológica contiene dos aspectos. El primero consiste en la reivindicación inequívoca, de inspiración aristotélica, de la animalidad (por más que sea especial) del hombre. En efecto, Aristóteles, con expresión sobria y sintética, presenta al hombre en diversos lugares como el animal racional o más exactamente como el animal que posee logos (zoón echón lógon). El segundo es que, contra la tradición de la clásica psicología racional, siguiendo la inspiración de Scheler en su obra señera La posición del hombre en el cosmos, el hombre es según la idea antropobiológica una síntesis ascendente de animalidad y racionalidad. La inspiración aristotélica es de nuevo evidente. A diferencia de la psicología racional, que desde Wolff en adelante privilegiaba el estudio del psiquismo superior (lo que ha sido llamado las zonas altas del hombre), dejando en la penumbra lo que eran los fundamentos biológicos del 
mismo, la idea antropobiológica se toma bien en serio el hecho de que el hombre es inicialmente, un animal que, como tal, se halla profundamente inserto en el mundo natural y que participa como ser orgánico (es decir, dotando de un cuerpo viviente) de una estrecha comunidad con los demás seres orgánicos que viven en el mundo (a saber, plantas y animales). La vieja psicología limitaba su estudio del hombre en lo fundamental a las funciones psíquicas superiores del hombre, sin tener suficientemente en cuenta que el tejado es sustentado por la casa y la casa por los cimientos. Este error de procedimiento es corregido en buena medida por la naciente antropología fenomenológica de la primera mitad del siglo XX, y especialmente por Scheler y Plessner con sus respectivas obras La posición del hombre en el cosmos y Los grados de lo orgánico y el hombre: introducción a la antropología filosófica.

En segundo lugar en este trabajo, como hemos dicho, pretendemos también echar mano de la historia para poner de manifiesto asimismo que la idea antropobiológica es todo menos moderna. Para ello creemos de suma utilidad trazar un bosquejo de algunas teorías antropológicas de determinados autores que, a lo largo de la historia, han asumido inequívocamente la idea antropobiológica. En tal sentido propondremos algunas ideas de Aristóteles, Tomás de Aquino, Kant, Herder, Uexküll y Lorenz.

\section{La idea antropobiológica}


Leopoldo Prieto

\subsection{Una nueva valoración de la corporalidad humana}

Junto a la manifiesta confusión que reina en la cuestión hombre-animal, existe en nuestros días un interés creciente por los animales, pero sobre todo por conocer mejor aquello que el hombre comparte con ellos. Este interés en el conocimiento de lo común al hombre y al animal no es algo nuevo. El viejo esquema de los grados de vida, a saber, vegetal, animal y racional, admitía con toda naturalidad que en el animal hay estratos de vida vegetativa, así como en el hombre se encuentran también los niveles vegetativo y sensitivo (o animal). La idea aristotélica del hombre como animal racional es inequívoca en este sentido. Pero es verdad que el esquema de los grados de vida, al menos en lo que se refiere al estudio del hombre, propició un desinterés y un olvido, especialmente en la modernidad, de la corporalidad y sensibilidad humanas. En este contexto hemos asistido en el siglo XX a un interesante y prometedor cambio de orientación en la antropología. Las antropologías biológicas se han caracterizado por afrontar el estudio del ser humano desde una perspectiva que, aunque es propiamente filosófica, modifica profundamente la orientación de los precedentes estudios sobre el hombre. El cambio de perspectiva adoptado, que podría considerarse una suerte de revolución copernicana de la antropología, ha consistido en plantear el estudio filosófico del hombre desde un nuevo punto de partida que centra su atención inicialmente en el cuerpo humano.

La fecundidad de la nueva perspectiva ha quedado acreditada ante todo con el descubrimiento en el cuerpo humano de una serie de rasgos físicos atípicos e inexplicables a la luz de zoología. Estos rasgos físicos proporcionan 
los datos necesarios de los que se valen las antropologías biológicas para concluir (en un sentido filosófico naturalmente, como es propio de su método) que el cuerpo humano es el correlato físico de una criatura racional. La ilimitada apertura de la razón humana a la realidad tiene su reflejo en la inespecialización morfológica del cuerpo humano, que aparece como un cuerpo abierto, es decir carente de especialización (aunque por ello mismo más vulnerable físicamente), desvinculado del ambiente físico y libre de las ataduras que el medio ambiente impone a la morfología de cualquier animal. Asimismo, la ilimitada apertura de la voluntad (que es el fundamento profundo de la libertad), tiene una correspondencia análoga en la indeterminación física de la conducta humana. En definitiva, las carencias humanas tanto de especialización morfológica como de instintos animales hacen del hombre un ser biológicamente anómalo y un animal indigente. En este sentido decía Nietzsche que "el hombre es un ser más enfermo, más inseguro, más cambiante y menos definido [kränker, unsicherer, wechselnder, unfestgestellter] que cualquier otro animal", de manera que "no hay duda que es el animal enfermo [kranke Tier]" (Nietzsche, 1892, 129).

\subsection{Antropología biológica y fenomenología}

Como se ha dicho antes, a lo largo del siglo XX se ha desarrollado un nuevo tipo de antropología filosófica que se puede llamar biológica. Esta escuela antropológica, si es lícito considerarla así, está particularmente relacionada con la fenomenología. De hecho sus autores más importantes pertenecen también de alguna manera a la fenomenología. 
Ciertamente uno de los mayores méritos de la fenomenología ha sido el haber dado vida a la actual antropología filosófica, separándola de la clásica psicología racional, especialmente con la obra de Max Scheler, El puesto del hombre en el cosmos (1927). Desde el momento mismo de su nacimiento, esta nueva antropología filosófica se ha interesado constantemente por el estudio de lo común, primero, y de lo específico, después, del hombre frente al animal. En este sentido, algunos de los iniciadores de la antropología filosófica (Scheler, Plessner, Gehlen, etc.) son considerados justamente pioneros de la idea antropobiológica. No vamos a hablar de ellos en este trabajo. Pero sí de algunos autores, filósofos y científicos, en los que estos fenomenólogos han encontrado información e inspiración para la nueva orientación de la antropología que han hecho posible.

Las antropologías biológicas, como cualquier empresa humana, no han carecido de algunos defectos. En mi opinión el más obvio es un cierto tono antimetafísico, que comparte con la mayor parte de las corrientes de la antropología filosófica del siglo XX. Pero tienen algunos méritos considerables que dan un neto carácter positivo al balance general de este nuevo enfoque antropológico y hacen verdaderamente interesante su estudio. Si en su gran mayoría las antropologías del siglo XX han negado la existencia de la naturaleza humana, un mérito sin duda no pequeño de las antropologías biológicas ha consistido en redescubrir y proponer de un modo nuevo el concepto de naturaleza humana, inducido en parte por contraste (o en comparación) con la naturaleza del animal. En este redescubrimiento de la naturaleza humana, estudiando las obvias diferencias físicas entre el hombre y los demás anima- 
les, las antropologías biológicas han puesto de manifiesto no sólo los caracteres específicos del cuerpo humano frente al del animal, sino que, a la vista de los caracteres somáticos del ser humano, han llegado a la conclusión de que el cuerpo humano es un cuerpo atípico según las exigencias de la zoología. Este aspecto, conocido en realidad desde siempre, pero caído en olvido en los últimos siglos (probablemente por influjo del racionalismo y dualismo modernos), constituye una aportación de la antropología biológica de indudable valor. Es una paradójica conquista: insistiendo en el estudio de los aspectos físicos del ser humano, han salido a la luz valiosas observaciones sobre la inteligencia, la voluntad, la racionalidad, y en definitiva sobre el espíritu, sin el cual la criatura humana, dotada de un cuerpo de una anómala indigencia biológica, no habría logrado sobrevivir.

Así pues, partiendo no directamente del estudio del alma racional, como clásicamente hacía la psicología racional, sino del cuerpo humano, de su morfología y de sus peculiares disposiciones, las antropologías biológicas han encontrado un considerable número de datos empíricos de gran valor que apuntan a la espiritualidad del ser al que pertenece un cuerpo tan peculiar desde el punto físico. De este modo, el espíritu se convierte en la clave de interpretación profunda de la naturaleza humana, incluida su componente somática. En tal sentido el biólogo suizo A. Portmann escribió un libro cuyo título Biología y espiritu (Biologie und Geist) evoca esta idea. La observación de la precariedad biológica del cuerpo humano es una constante a lo largo de la historia del pensamiento. Platón, Aristóteles, Cicerón, Agustín de Hipona, Tomás de Aquino, Hobbes, Kant, Herder, además de otros muchos, la 
conocían perfectamente y la hicieron frecuentemente objeto de inteligentes comentarios en sus obras. Pero nunca se había convertido en tema de estudio sistemático. Y tanto menos había sido hecha punto de partida en el estudio del hombre.

Se puede decir, por tanto, que la idea central de las antropologías biológicas es que el hombre es un ser en cuyo cuerpo, y no sólo en su inteligencia y voluntad, se hace patente la presencia de la racionalidad (o del espíritu). La apertura es propia de las entidades espirituales. Ahora bien, la apertura del ser humano a la realidad no es una propiedad exclusiva de su razón. Todo el ser humano, también su cuerpo, participa de algún modo de esta característica. El cuerpo humano manifiesta unos rasgos tales que constituyen un auténtico desafío epistemológico para la zoología y que son el reflejo tanto en la morfología corporal como en el comportamiento de la característica universalidad racional. Así ha de entenderse la carencia, típicamente humana, de adaptación al medio ambiente, es decir, la inespecialización morfológica. También a esta luz ha de considerarse la ilimitada apertura del comportamiento humano, como es propio de un ser que, careciendo de instintos, debe guiar sus acciones mediante la razón y la libertad.

Conviene dejar claro desde el inicio que el principio fundamental de las antropologías biológicas no es constatable experimental, sino filosóficamente. La realidad del espíritu no puede ser aprehendida con los métodos y procedimientos aplicados por la ciencia experimental al estudio de la realidad física, que se limita al tratamiento cuantitativo de realidades de índole material. Tal tipo de ciencia deja por principio fuera de su campo visual las 
dimensiones no cuantificables, es decir, no materiales, de la realidad. La ciencia es incompetente, por definición, en todo lo que se refiere al espíritu. Éste es una realidad que escapa a las exigencias del objeto y del método de la ciencia experimental. Pero el espíritu es la dimensión esencial del ser humano. Por eso, la aportación de la filosofía a este campo es no sólo preciosa, sino indispensable. De ahí la necesidad de cooperación interdisciplinar entre biologia y filosofía. En cualquier caso, creemos que la ciencia debe admitir que no es la única forma posible de racionalidad, dejando abierto el camino a otras formas de saber, como son la filosofía y la teología.

\subsection{Interdisciplinariedad de biología y filosofia en el estudio del hombre}

El propósito común a las diversas antropologías biológicas no es tanto teorizar sobre el hombre, sino indicar dimensiones poco conocidas en general de la naturaleza humana, que relacionadas con datos biológicos relevantes (señalados por biólogos y filósofos del siglo XX), ayudan a comprender mejor que el hombre es un ser en el que se constata una profunda unidad psicosomática, o lo que es igual, una unidad profunda de cuerpo y alma. De manera que el cuerpo bumano no es un cuerpo simplemente animal, sino bumano - valga la redundancia-, por lo que en él se manifiestan, aunque de un modo indirecto, como no podía ser otro modo, aspectos característicos de la racionalidad y del espíritu.

Las antropologías biológicas constituyen, pues, un intento de reflexión interdisciplinar entre biología (biología teórica, zoología y etología) y filosofía. La interdisciplinariedad, en una época de fragmentación de las ciencias, es el primer paso en la búsqueda de la recomposición de la unidad del saber, que es la 
aspiración de toda verdadera sabiduría. Sólo la sabiduría, que los clásicos entendían como ciencia de las causas últimas, puede concebir un plano general del saber, aunque sólo en sus trazos fundamentales, dada la natural limitación de la mente humana. La pérdida de esta unidad se ha debido fundamentalmente a dos causas: al desarrollo exponencial de las ciencias particulares en áreas muy delimitadas del saber, y también al progresivo abandono de la metafísica, la antigua regina scientiarum, única ciencia capaz —creemos- de proporcionar una teoría razonada de los géneros supremos de la realidad. Una teoría de los géneros supremos de la realidad no es otra cosa que una teoría de las categorias, que estudia los modos de ser fundamentales de la realidad (la sustancia y los diversos tipos de accidentes). Pues bien, la necesidad de la interdisciplinariedad es especialmente acuciante en el campo de los estudios sobre el hombre, donde una cantidad enorme de datos resulta cada vez más difícil de resolver en unidad. Ahora bien, sin unidad, no hay comprensión. Comprender algo significa ver simultáneamente, en la unidad de su naturaleza, la multiplicidad de aspectos que la integran. Por eso, resignarse al actual estado de fragmentación del saber antropológico es resignarse a desconocer qué es el hombre. La filosofía, como saber típicamente unificante, en la medida que es una sabiduría, puede ayudar a remediar en algo este estado de cosas. Pero necesita la información que sólo pueden proporcionarle las ciencias particulares sobre el hombre. En fin, pocos proyectos de reflexión interdisciplinar son tan urgentes como el de la cuestión antropológica. Precisamente para alcanzar este fin surgió la antropología filosófica en la primera mitad del siglo XX. Y precisamente por esta razón ha sido la misma antropo- 
logía filosófica la ciencia que ha estimulado y fomentado la cooperación de las ciencias afines o colindantes con la antropología, sobre todo la biología, para hacer luz en la magna cuestión del hombre.

\section{Breve historia de la idea antropobiológica}

Ha sido mérito de los filósofos haberse percatado, antes que los científicos, de las características humanas de inespecialización orgánica e inadaptación ambiental.

\subsection{Platón}

Platón parece haber sido el primero en notar estas características humanas. En el mito de Prometeo, narrado en el Protágoras, un relato alegórico sobre la creación del hombre en el que abundan las intuiciones profundas, nos presenta Platón al hombre como un ser que, a diferencia de los demás animales, carece de la mayor parte de las facultades orgánicas de estos por causa de la ineptitud de Epimeteo, que fue el encargado de realizar la distribución de los dones naturales el día fijado por el destino para su creación. Prometeo, por su parte, para remediar esta situación de penuria orgánica en que el hombre había quedado, entregó a éste, después de haberlos robado a Hefesto y Atenea, el saber técnico y el fuego, con cuya posesión quedaba ampliamente compensada (ahora en beneficio del hombre frente a los demás animales) la pobreza orgánica que lo aquejaba. De manera que en esta nueva situación el hombre pasa a superar a los demás animales hasta el punto de que, como 
sugiere Platón, sólo el hombre "participa en el dominio divino", porque ha recibido el parentesco con la divinidad misma. Sin embargo, esta grandeza del hombre no impide que la vida en las ciudades de los hombres, recién erigidas, se vea amenazada continuamente por la destrucción de las guerras. Por ello Zeus se ve en la obligación de enviar a Hermes, su emisario, para que restablezca la justicia y enseñe a los hombres la ley de Zeus, es decir, la virtud y la amistad recíproca entre los hombres, únicos fundamentos sobre los que puede descansar la vida humana en paz y prosperidad ${ }^{1}$.

\subsection{Aristóteles}

Pero no fue Platón el único en constatar la peculiar configuración orgánica del hombre. Otros filósofos también han llamado la atención sobre esta condición especial del hombre. Entre ellos destaca Aristóteles. Si Platón pone el acento en la penuria orgánica del ser humano, Aristóteles llamaba la atención sobre la inespecialización orgánica del mismo. En concreto, en Las partes de los animales estudia Aristóteles la función y estructura de la mano, llegando a la conclusión de que ésta no es instrumento para algo, sino un instrumento de instrumentos, dado a la inteligencia humana como medio de ejecución de sus planes. La clave, pues, de la idea antropobiológica en Aristóteles es la idea de apertura cognoscitiva de la inteligencia y de apertura ejecutiva de la mano, o en última instancia, la no determinación ad unum de ambas. De este modo la conjunción de inteligencia y mano pone remedio a la

1 Cf. Platón, Protágoras 320 c - 322 d 
pobreza física del ser humano ya advertida por Platón. Dice Aristóteles a este respecto:

Razonan incorrectamente aquellos que sostienen que el hombre no ha sido bien formado o que incluso lo ha sido peor que el resto de los animales, pues, añaden, el hombre no tiene protección en los pies, está desnudo y desprovisto de armas de combate. Los otros animales tienen un solo medio de defensa y no les es posible sustituirlo con ningún otro, sino que deben dormir y hacer cualquier otra cosa teniendo siempre, por así decir, los zapatos en los pies, es decir, sin poderse desprender de la coraza que tienen en el cuerpo, ni pueden cambiar el arma que les ha tocado en suerte. Al hombre, en cambio, han sido concedidos muchos medios de defensa, pudiendo siempre cambiarlos y adoptar el arma que quiere en el momento que quiere. La mano, en efecto, puede convertirse en garra, tenaza, cuerno, lanza, espada o cualquier otra arma o instrumento, ya que puede aferrar y empuñar todas estas cosas².

Aristóteles deja incluso entrever la analogía entre la mano, calificada como instrumento de instrumentos, y el intelecto, que es forma de las formas ${ }^{3}$.

Estando naturalmente en posición erecta, el hombre no necesita las patas anteriores, en cuyo lugar la naturaleza lo ha provisto de brazos y de manos. Por eso Anaxágoras afirma que el hombre es el más inteligente de entre los animales, por el hecho de poseer las manos. Pero en realidad lo razonable es decir que ha obte-

2 Aristóteles, Partes de los animales, IV, 10, 687 b 1-27

3 Cf. Aristóteles, Sobre el alma, III, 7, 431 b 28 
nido las manos porque es el más inteligente [...] El más inteligente debe ser el que es capaz de servirse de un mayor número de instrumentos. Ahora bien, la mano parece ser no uno, sino muchos instrumentos. En cierto sentido la mano es un instrumento para usar otros instrumentos ${ }^{4}$.

Un interesante estudio de la teoría aristotélica sobre la naturaleza humana ha sido realizado recientemente por J. Benéitez. Palabra y polis son en su opinión los dones que elevan a la naturaleza humana sobre la animal (Benéitez, 2011, 16-24).

\subsection{Tomás de Aquino}

Siglos más adelante, Tomás de Aquino recibe las enseñanzas de Aristóteles. Se expresa por ello en términos semejantes, pero añade algunas interesantes observaciones sobre las carencias orgánicas del hombre y la infinitud potencial que la razón abre al hombre. Hablando del alma intelectual, dice a modo de objeción en la Suma teológica:

El alma intelectual es la más perfecta de las almas. Así pues, como quiera que los cuerpos de los otros animales tienen naturalmente con qué protegerse, como pelo en lugar de vestido y pezuñas en lugar de calzado, y además tienen armas dadas por la naturaleza, como uñas, dientes y cuernos, parece que el alma intelectual no debería unirse a un cuerpo imperfecto [como es el humano] desprovisto de tales ayudas (Tomás de Aquino, 2001, 694).

4 Aristóteles, Partes de los animales, IV, 10, 687 a 
A lo cual responde el propio autor más adelante, estableciendo explícitamente la relación entre pobreza biológica y espiritualidad del ser humano, en los siguientes términos:

$\mathrm{El}$ alma intelectual, porque puede comprender lo universal, tiene capacidad para lo infinito. Por eso la naturaleza no podía imponerle determinadas estimaciones naturales, ni tampoco determinados medios de defensa o de abrigo como a los otros animales cuyas almas tienen capacidad de percepción y otras facultades para cosas particulares. Pero en su lugar, el hombre posee por naturaleza la razón y las manos, que son órgano de los órganos, por medio de las cuales puede preparar una variedad infinita de instrumentos para infinitos efectos (Tomás de Aquino, 2001, 695).

Comentando en otra obra el texto del De Anima de Aristóteles, arriba citado, dice Tomás de Aquino que "el alma es comparable a la mano", pues así como el alma intelectual es forma de las formas (es decir potencia receptiva virtualmente infinita para concebir toda la realidad), del mismo modo

...la mano es órgano de órganos, habiendo sido dada al hombre en lugar de los demás órganos que son dados a los animales para la defensa, el ataque, o el vestido, cosas todas éstas que el hombre se prepara por medio de la mano (Tomás de Aquino, 1959, 13). 


\subsection{Kant}

También en diversos pasajes de la obra de Kant se encuentran observaciones que podríamos considerar de interés para esta breve presentación histórica de la idea antropobiológica. Tanto en la Pedagogía, como en el Probable inicio de la historia humana y en las Ideas para una historia universal en clave cosmopolita se encuentran afirmaciones de interés a nuestro propósito.

En la Pedagogía afirma Kant:

El hombre es la única criatura que ha de ser educada [...] Un animal lo es ya todo por su instinto. Una razón ajena lo ha provisto de todo. Pero el hombre necesita una razón propia. No tiene ningún instinto y ha de construirse él mismo su plan de conducta (Kant, 1962, 441).

De manera que, así como al hombre ha sido dada la razón, se le ha privado de instintos. "La naturaleza no da dado al hombre instinto alguno" (Kant, 1962, 447).

En el Probable inicio de la historia humana afirma Kant que, habiendo adquirido el hombre la razón, se separó del modo de vida de los animales, que es invariablemente regido por el instinto. Descubrió entonces en sí la capacidad de elegir y determinarse un propio camino de conducta, más allá de la estrechez que caracteriza la vida de los animales. Pero con el uso de la razón descubrió también la esperanza del futuro, tanto de los proyectos venideros a realizar como de la inevitable muerte a padecer, que sólo el hombre conoce como tal. 
$\mathrm{El}$ instinto $[\ldots]$ al que obedecen todos los animales, era lo único que guiaba inicialmente al hombre inexperto [...] Mientras el hombre obedeció a esa llamada de la naturaleza, se encontró a gusto con ello. Pero en seguida la razón, comenzó a despertarse dentro de él (Kant, 1969b, 111).

De este modo el hombre cobra conciencia de que la razón lo capacitaba para sobrepasar los límites ante los que se detienen todos los animales. Descubre así la capacidad de elegir por sí mismo su propia manera de vivir y no estar sujeto a una sola forma de vida, como el resto de los animales (Cf. Kant, 1969b, 112).

Descubre también así la expectativa de futuro, que es la capacidad de hacerse presente un tiempo por venir, incluso muy lejano, lo que permite al hombre trabajar por fines muy remotos con arreglo a su naturaleza; pero al mismo tiempo es una fuente inagotable de preocupaciones y aflicciones que suscita la incertidumbre del futuro, de las que están exentos todos los animales [...] También anticipa con temor, como telón de fondo de una vida tan fatigosa, algo que sin duda también afecta a todos los demás animales, pero que no les preocupa en absoluto: la muerte (Kant, 1969b, 113).

Finalmente, en las Ideas para una historia universal en clave cosmopolita insiste Kant en la vieja idea de la penuria orgánica que caracteriza la vida física del hombre.

El hombre [en cuanto dotado de razón y libertad] no debía ser dirigido por el instinto o instruido por conocimientos innatos, antes bien debía extraerlo todo de 
Leopoldo Prieto

sí mismo. La invención de sus productos alimenticios, de su cobijo, de su seguridad y defensa exteriores (para lo cual la naturaleza no lo dotó de los cuernos del toro, de las garras del león, ni de la dentadura del perro, sino de simples manos), todo deleite que pueda hacer grata la vida, hasta la inteligencia y astucia [...] debían ser enteramente obra suya. De este modo la naturaleza parece haberse complacido en dotarlo del modo más pobre y en haber adaptado su equipamiento animal de un modo muy escaso y ajustado a la máxima necesidad de una existencia inicial (Kant, 1969a, 19-20).

\subsection{Herder}

Sin embargo, el precursor más lúcido de la idea antropobiológica es Herder. En su obra Ensayo sobre el origen del lenguaje (1772) se propone por primera vez una teoría de los rasgos diferenciales del hombre frente al animal.

Afirma Herder, en primer lugar, que "está fuera de duda que el hombre se halla a mucha distancia de los animales en lo que toca a la fuerza y seguridad del instinto, que incluso carece en absoluto de lo que llamamos en muchas especies animales facultades y tenencias innatas" (Herder, 1982, 145-146). Cuál es la verdadera causa de la carencia de instintos en el hombre - dice Herder- es algo que todavía no se ha podido clarificar. Herder ha entrevisto también la importancia decisiva de la noción de mundo circundante, a la que da el nombre de esfera de los animales (Sphäre der Tiere), para comprender la conducta animal. Cada animal, nos dice, se desenvuelve dentro de una determinada esfera, a la que pertenece desde su nacimiento hasta la muerte. Ahora bien, es curioso que cuanto más agudos son los sentidos de los anima- 
les y cuanto más precisas son sus acciones, tanto más pequeña y limitada es la esfera en la que viven (Herder, 1982, 146).

Tras estas averiguaciones relativas al instinto y al mundo circundante del animal, Herder sugiere que la tendencia fundamental en el modo de vida animal puede ser formulada en los siguientes términos: existe una proporción inversa entre la precisión de los sentidos y de los instintos animales y la amplitud de la esfera en la que se desenvuelve su vida.

La abeja construye su colmena con una sabiduría que Egeria no era capaz de enseñar a Numa. Pero fuera de sus celdas y de su predeterminada labor en ellas, no es nada. La araña teje con el arte de Minerva, pero todo su arte se halla encerrado en el limitado espacio de tela. Ése es su mundo. ¡Que admirable es el insecto y qué estrecho es su círculo de acción! (Herder, 1982, 146).

Un siglo más tarde Jean-Henri Fabre (1823-1915) confirma estas apreciaciones en su monumental obra Souvenirs entomologiques (10 vols., 18791907), considerada la fundación científica de la entomología. Se puede formular esta tendencia en los siguientes términos: cuanto más limitada es la esfera de un animal, tanto más seguras y perfectas son sus acciones; y cuanto más amplia es su esfera vital, es decir, cuanto más variadas son sus funciones, cuánto más abierta es la atención sobre diversos objetos, cuanto más inestable y menos fijo es su modo de vida, tanto más débil y dividida resultan su sensibilidad y sus instintos (Cf. Herder, 1982, 146). En una palabra, "la sensibilidad, las facultades y los instintos de los animales incrementan su 
fuerza y perfección en proporción inversa a la extensión y variedad de su círculo de acción” (Herder, 1982, 147).

Así se comprende que el animal, dotado de sentidos inmensamente más finos y precisos que los del hombre, permanece por ello mismo clausurado en un pequeño círculo y en una única dirección, mientras que el resto del mundo carece por completo de significación para él. Por esta razón las acciones del animal son tan certeras y atinadas. Y a la inversa: cuanto más variadas son las funciones a las que el animal está destinado; cuanto más se amplía su atención a diversos objetos; cuanto más inquieto es su modo de vida; en una palabra, cuanto mayor y más variada es su esfera, exactamente como ocurre en el caso del hombre, tanto más se fragmenta y se debilita su capacidad sensorial.

En comparación con el animal, el hombre se caracteriza por sus deficiencias.

El niño recién nacido es, en comparación con los animales, la criatura más desamparada de la naturaleza: desnudo, débil, necesitado, temeroso y desarmado. Y lo que lo hace la suma de la pobreza: desprovisto de toda guía en la vida. Con una capacidad sensorial tan pobre, con facultades de tal modo indeterminadas y potenciales, con pulsiones tan fragmentarias y desvaídas [...] destinado a vivir en medio del gran círculo (Herder, 1982, 149).

De todo esto, en comparación con el animal, la descripción más adecuada del hombre es negativa, dado que su nota específica consiste en la privación y en las carencias. 
Historia de la idea antropobiológica

Sus sentidos y su organización no operativa no tienden a una cosa sola. Tiene sentidos para todo, y por ello mismo sus sentidos son débiles y obtusos para cada cosa en particular. Las fuerzas de su alma están repartidas por todo el mundo. Sus ideas no se dirigen hacia una cosa sola. Por eso carece de pulsiones y actitudes terminadas y definidas (Herder, 1982, 149).

En una palabra, el hombre carece de mundo circundante, del circulo estrecho de los animales en el que las operaciones están instintivamente prefijadas. "Ante el hombre se abre un mundo de asuntos y determinaciones. Sus apetencias están dispersas, su atención dividida, sus sentidos entumecidos" (Herder, 1982, 147).

Estas notas nos bastan para hacernos una idea de la orientación de la antropología de Herder. Su gran mérito está sin duda en haber puesto de manifiesto con rara lucidez la estrecha relación que hay entre la constitución biológica humana, determinada por sus carencias biológicas, y su específica apertura al mundo. Con ello queda dicho, al menos entre líneas, que entre el hombre y el animal no hay que buscar diferencias de grado, en el sentido de un mayor desarrollo humano. Lo que ocurre es justamente lo contrario: el mayor desarrollo y especialización orgánicos son los del animal. Las diferencias, pues, entre el hombre y el animal pertenecen a otro orden de cosas. Es todo el hombre el que se diferencia del animal: tanto en su alma, que es racional y no simplemente sensitiva, como en su cuerpo, que es un cuerpo atípico. Pero tales diferencias proceden de una raíz más profunda. $Y$ es que el hombre es tal, corporal y espiritualmente, porque se halla implantado en un 
nuevo nivel de realidad, esencialmente oculto (y superior, claro está) al nivel en que se encuentra el animal. A este nuevo nivel de realidad lo llamamos mundo. Ahora bien, la implantación en el mundo sólo es posible a aquellos seres dotados en su conocimiento y en sus apetitos de una amplitud y de apertura tan vastos como el mismo mundo. En una palabra, hallarse implantado en el mundo significa ser una criatura espiritual. Una criatura espiritual es aquella cuyo conocimiento y voluntad se hallan potencialmente abiertos a la totalidad de lo real, es decir a todo lo real en cada cosa y a todas las cosas reales.

A este modo de implantación en la realidad, esencialmente diverso del propio del animal, debido a una constitución ontológica de índole espiritual, se debe la profunda diferencia que se percibe fácilmente entre las facultades del hombre y del animal. En el hombre el conocimiento termina en la razón y los apetitos son coronados por la libertad. En el caso del animal, en cambio, el conocimiento se agota en el sentido y el apetito en el instinto. Todas las dimensiones de la realidad humana apuntan hacia el nuevo ámbito de realidad solo accesible a la razón y la libertad. De ahí que la agudeza de sus sentidos y la correspondiente seguridad, en términos biológicos, de su conducta sean siempre inferiores a las del animal. Mayor destreza orgánica, mayor precisión sensorial, mayor determinación operativa serian tanto como mayor animalización del hombre, mayor adaptación al medio, mayor limitación a un círculo vital cerrado. Que el hombre es biológicamente inferior al animal es una exigencia de su espiritu. Herder lo ha visto con agudeza inigualable. Efectivamente, hay una proporción inversa entre la amplitud del campo de realidad y la precisión 
y riqueza del aparato sensorial y motriz de que están dotados hombres y animales. En el caso del hombre la distancia entre los términos de esta proporción inversa es la mayor pensable.

La naturaleza ha intentado con el hombre el proyecto de un ser viviente completamente nuevo y diverso de las demás criaturas animales. En palabras del propio Herder:

El hombre tiene el privilegio real de mirar lejos a su alrededor con la cabeza alta y erguida [...] Gracias a su destinación a la razón, no concedida a ninguna otra criatura terrena, el hombre es progenie divina y rey de la tierra [...] La elevación del destino humano se hace presente en los dos grandes dones, razón y libertad, que la Naturaleza ha osado confiar a una criatura terrena tan débil como es el hombre. El animal es solamente un esclavo inclinado sobre el suelo [...] Su alma, inmadura para la razón, tiene que servir a sus instintos [...] El hombre es el primer ser de la creación dejado en libertad. Por ello está erguido. La balanza del bien y el mal, de la verdad y del error, está en sus manos. El hombre puede buscar. El hombre tiene que elegir (Herder, 1994, 146, trad. propia).

\subsection{Von Uexküll}

Ya en el siglo XX el primer estudioso promotor de la idea antropobiológica ha sido Jakob von Uexküll. La idea central de su pensamiento es que a cada animal, o mejor a cada especie animal, corresponde un espacio biológico propio, también llamado mundo circundante (Umwelt), constituido por la misma organización biológica del animal, que selecciona, de entre todas las propiedades y características de las cosas y animales existentes a su alrededor, 
solamente aquellas de interés para su vida. El espacio biológico o mundo circundante no es la suma de cosas que circundan físicamente al organismo, sino el conjunto de aquellas características ambientales que desempeñan una función biológica o que tienen una significación vital para el animal. Las restantes propiedades ambientales forman un conjunto inobservado de información que el animal ignora, ya que no pasan a través del filtro de la percepción sensorial y tampoco causan reacciones en el animal que se encuentra frente a ellas. Únicamente por su inserción en el mundo circundante las cosas adquieren una significación (Bedeutung) para el animal, aunque de naturaleza exclusivamente biológica. Todo lo que de facto excede los confines de este campo biológico, aunque esté físicamente presente, no existe para el animal. En cambio, en el interior de este campo biológico, que es el llamado mundo circundante, las cosas y los animales se hacen portadores de un tono (Tön) significativo que les permite entrar en relación con las disposiciones biológicas del animal, el cual a su vez se encuentra fisiológica y funcionalmente predispuesto a reconocer tal significación y a obrar conforme a ella.

\subsubsection{Mundo circundante}

Ya hemos hablado del entorno biológico o mundo circundante dentro del cual cada animal no sólo vive, sino que incluso construye. Pues bien, según von Uexküll, dentro de su ámbito vital cada animal se comporta como un sujeto, porque no es un ser inerte sino activo. La actividad del animal, aún del más ínfimo y pobremente dotado, como en el caso de los animales unicelulares, consiste esencialmente en darse cuenta de algo y actuar consecuentemente con lo percibido. Mediante esta actividad vital el animal 
construye su mundo circundante. De ahí, concluye von Uexküll que el mundo circundante de cada animal no es sino esto: un mundo de señales accesibles al animal, del que procede un conjunto bien determinado de acciones y pautas de comportamiento. El mundo de señales y el mundo de impulsos forman una unidad funcional cerrada. A su vez, los mundos circundantes propios de cada animal difieren entre sí tanto como los animales mismos, de cuya actividad cognoscitiva y operativa proceden (von Uexküll, 1956, 26-27).

\subsubsection{Significación}

El segundo principio de la biología de Jacob von Uexküll es la significación. Las cosas y los demás animales representan algún interés para el animal sólo en la medida en que pueden despertar en él un estímulo y suscitar alguna de las acciones contenidas en su repertorio de respuestas. Si un objeto se demuestra incapaz de suscitar algún tipo de respuesta en el animal, aquél, careciendo de interés para éste, es abandonado como si no existiera. Pues bien, las propiedades que hacen de un objeto (cosa o animal) algo biológicamente de interés para un animal en cuestión constituyen lo que von Uexküll llama significación. La significación es, por tanto, la correspondencia o proporción del objeto con la dotación cognoscitiva y operativa del animal. Como el interés suscitado en el animal no puede proceder más que de esta correspondencia del objeto con sus percepciones y posibles comportamientos, es claro que la significación presupone el mundo circundante. Así pues, todo objeto contenido dentro del mundo circundante de un animal es necesariamente significativo para él; y por ello mismo es portador de señales tanto receptivas o de cono- 
cimiento (Merkmalträger) como de señales suscitadoras de una reacción (Wirkmalträger).

\subsubsection{Círaulo funcional}

A su vez, las relaciones significativas que se traban dentro del mundo circundante adoptan la forma de un círculo vital, al que von Uexküll llama circulo funcional (Funktionskreis). Las relaciones entabladas entre el animal y el objeto significativo se pueden describir adecuadamente siguiendo el esquema del círculo funcional, al modo de preguntas y respuestas. El círculo funcional presupone, por tanto, que en el animal existe un ámbito interior, un mundo interior como lo llama nuestro autor, del cual, después de recibirse una determinada información, procede la correspondiente respuesta adecuada a los intereses biológicos del animal. En razón de este mundo interior (que no es otro sino lo que Aristóteles llamaba el alma sensitiva), el animal es un viviente, esencialmente distinto de cualquier ser inerte, dotado de una serie de operaciones propias, como son: el conocimiento sensible, que le permite seleccionar la información de interés (o significativa), y la propensión instintiva, con la que responde adecuadamente a la satisfacción de sus necesidades.

El círculo funcional se asemeja, para tomar una imagen sencilla, a los dos brazos de unas tenazas (von Uexküll, 1956, 26-27). Así pues, cada animal entra en relación con su objeto por medio de estos dos brazos: el brazo de la observación (Merkglied) y el brazo de la efectuación (Wirkglied). Con el primero advierte algo del objeto (aquello cuya significación despierta su interés), que se presenta con el carácter de noticia (Merkmal); con el segundo brazo, el de la 
efectuación (Wirkmal), lleva a cabo, de entre las posibilidades que constituyen su repertorio de posibles acciones, la ejecución adecuada al objeto a modo de respuesta. De este modo, determinadas propiedades del objeto se transforman en portadoras de señales receptivas, y otras, en portadoras de señales efectivas. Como todas las propiedades radican y se hallan juntas en la unidad misma del objeto, las propiedades percibidas como señales efectivas tienen que ejercer su influjo sobre las propiedades que llevan el carácter de señales receptivas, dando por cerrado el círculo y disponiéndose para la apertura de otro nuevo. Brevemente se puede expresar así: la señal efectiva extingue la señal receptiva y da origen a un nuevo círculo funcional.

\subsubsection{Finalidad}

El concepto de círculo funcional sugiere de este modo la unidad profunda que se establece entre el animal y su objeto. La estrecha unidad e interdependencia que se advierte entre las propiedades del objeto significativo y las del animal, con una concordancia y correlación en verdad sorprendentes, hace pensar a von Uexküll en la idea del punto y del contrapunto de una melodía (von Uexküll, 1940, 149). Esto lleva a decir a nuestro autor que "hay algo en las abejas que es para las flores, así como hay algo en las flores que es para las abejas" (von Uexküll, 1940, 145).

Si del ejemplo se pasa al nivel de los principios, se podría hablar con von Uexküll del contrapunto en biología como causa de la formación de los animales (von Uexküll, 1940, 145). Si el contrapunto musical consiste en la sobreposición de varias líneas melódicas, el contrapunto en la biología da a conocer la admirable ordenación de las partes que componen la naturaleza; 
en nuestro caso, de la perfecta adaptación del animal a su medio. La tela de araña está hecha en vista de las moscas, porque la araña misma está en relación vital con las mismas. Y estar en relación vital con las moscas quiere decir que la araña en su misma estructura biológica ha adoptado determinados elementos en vista de las moscas. Algo parecido ocurre con la adopción de determinadas propiedades de los mamíferos en relación con la estructura biológica de la garrapata o del murciélago con la formación del oído interno de la mariposa nocturna. "Por todos lados en la naturaleza el contrapunto constituye el motivo para la conformación del animal" (von Uexküll, 1940, 146). Esta correspondencia admirable del animal con su hábitat es lo que von Uexküll ha llamado la "conformidad a un plan" (Planmässigkeit).

De aquí deduce nuestro autor el primer principio de la teoría del mundo circundante, a saber: que todos los animales, desde los más simples hasta los más complejos, se encuentran con idéntica perfección adaptados a su mundo. Adaptación y mundo circundante son nociones perfectamente correlativas. Al animal de constitución simple corresponde un mundo circundante simple; al más complejo pertenece un mundo circundante más rico.

\subsubsection{La significación biológica y significación objetiva: corolarios}

Pero no nos perdamos en detalles: para los animales (también los más desarrollados desde el punto de vista psicológico), lo que no entraña para su naturaleza una significación vital, no existe, o más exactamente, existe como algo que hay que dejar atrás. Así pues, la significación de cualquier cosa para el animal se limita a aquello que despierta un interés de naturaleza biológica, que concierne a la conservación y transmisión de su vida y a las conductas 
instintivas relacionadas con ello. Por ello esta significación puede ser llamada significación biológica.

Para el hombre, por el contrario, además de ésta significación que comparte con los animales, existe un campo de significado superior, que es de naturaleza lógica u objetiva. Por medio de la primera forma de significación el animal conoce de una cosa solamente aquello que puede entrar en relación con alguna de las necesidades que constituyen su repertorio de acciones vitales, que en lo esencial se limita a su conservación y reproducción. Mediante la segunda forma de significación, el hombre, en cambio, conoce qué es una cosa en sí misma, independientemente de cuánto pueda derivarse para él de provecho o de perjuicio de su trato. Se encuentra justamente aquí uno de los aspectos más importantes de diferenciación entre el hombre y el animal. El conocimiento sensitivo, el único del que dispone el animal, por más preciso y perfecto que sea (conviene no olvidar que muchos animales tienen un conocimiento sensitivo mucho más fino y desarrollado que el del hombre), se limita siempre a funciones de conservación y defensa de la vida. En cambio, la inteligencia, el conocimiento intelectual propiamente dicho, que es exclusivo del hombre, transciende el nivel meramente biológico y se implanta en un nuevo plano de la realidad, que es el de la esencia de las cosas.

\subsubsection{Primer corolario: principio del exdusivo interés biológico del animal en su mundo circundante}

Así pues, podemos ya formular sin más preámbulos un importante principio de la biología y de la antropología, a saber: que el mundo circundante del animal está completamente dominado por el interés biológico, es decir, por todo 
Leopoldo Prieto

aquello que es percibido como beneficioso o perjudicial para el propio animal o para su especie, con el inevitable comportamiento instintivo que lo conduce a procurárselo o a evitarlo. En el caso del hombre, por encima de la significación biológica, se abre un nuevo nivel de realidad: el campo inmenso de la realidad tal cual es, cuya significación sólo puede ser calificada de objetiva o lógica. Con ella el hombre conoce la esencia de las cosas, el modo de ser de cada una, en cuanto que es algo en sí misma, independientemente del beneficio que le pueda reportar o del daño que le pueda causar. Y, porque el hombre conoce las cosas, puede nombrarlas. Se contiene una profunda intuición en el Génesis cuando nos muestra al hombre pasando revista a las demás criaturas y dándoles a cada una un nombre, su nombre. No se equivoca el Génesis. Sólo el hombre, de entre todos los animales, puede nombrar las cosas, porque sólo él las conoce. El animal, en realidad, no conoce las cosas, sino en la escasa medida en que significan algo para él, es decir, en la medida en que le benefician o perjudican; o como dice von Uexküll de otro modo, en la medida en que entran a formar parte de su mundo circundante, que no es sino el acotamiento y el recorte de la realidad en vista de sus necesidades perentorias e inaplazables para conservar su propia vida o la de su especie.

\subsubsection{Segundo corolario: principio del predominio de la acción sobre el conocimiento}

Por otro lado, si observamos atentamente el principio arriba enunciado, según el cual el mundo circundante del animal está completamente dominado por el interés biológico, se comprende sin dificultad por qué el mundo 
Historia de la idea antropobiológica

circundante de los animales es a la vez operativamente seguro y cognoscitivamente pobre. Dentro del mundo circundante, que es un marco muy reducido de suyo, el animal se comporta con innata exactitud, y esto es precisamente lo que suele denominarse instintivo (Gehlen, 1961, 17). Sería lógico pensar que en el caso del animal, de igual modo que ocurre al hombre, el conocimiento de la realidad también debe preceder y disponer a la conducta. Pero en realidad ocurre lo contrario. Es decir, un animal puede conocer tantos objetos diversos cuantas acciones puede ejecutar sobre ellos. Lo que significa que el animal es un ser abocado a la acción (biológicamente entendida) y destinado exclusivamente a comportamientos ejecutivos de interés para su vida. Se puede hablar en este sentido de la operatividad como una dimensión esencial de la vida animal, faceta ésta que podríamos denominar el principio del predominio de la acción sobre el conocimiento o principio de vida exclusivamente práctica.

\subsection{Lorenz}

Acabamos de ver que la psicología animal gravita enteramente en torno a la conducta y no tanto al conocimiento. Esta ha sido también una de las grandes aportaciones de Konrad Lorenz y la nueva ciencia fundada por él: la etología. En el animal el conocimiento se limita a ejercer la función de un estímulo que pone en marcha el comportamiento. La función del conocimiento en el animal queda, pues, circunscrita a notificar la presencia en el mundo circundante de algún objeto (cosa o animal) capaz de estimular una determinada actividad, pero sin llegar a obtener un verdadero conocimiento del objeto. En esto justamente consiste el instinto, en la conjunción de una activi- 
dad (hacia la que el animal se encuentra genéticamente predeterminado) puesta en marcha por un factor desencadenante o excitador.

Para cada especie animal hay un número determinado de estímulos desencadenantes que provocan un comportamiento típico en todos los individuos de la misma especie. La conducta alimenticia, sexual, agresiva, etc., de estos individuos se pone en marcha cuando se dan las circunstancias biológicamente significativas que propician cada tipo de comportamiento. Los estímulos desencadenantes están predeterminados genéticamente y dictan al animal algunas pautas fijas de conducta. En cierto sentido, lo que se entiende por especialización animal es el estrecho ajuste existente entre estímulos, receptores, efectores y objetos de los que parten los estímulos. Gracias a este ajuste logra el animal una perfecta adaptación tanto morfológica como de la conducta a su mundo circundante. Asimismo, el ambiente en el que vive el animal se corresponde con esa especialización. El mundo circundante consta, ante todo, del sistema de estímulos capaces de provocar las conductas o movimientos instintivos propios de la especie. Entre mundo circundante e instinto, por tanto, hay una estrecha correlación.

En opinión de K. Lorenz el instinto animal consiste en la unión de dos elementos de naturaleza diversa, a saber: los estímulos o mecanismos desencadenadores innatos (MDI) y los movimientos instintivos. Ambos elementos son procesos fisiológicamente independientes. El mecanismo desencadenante es el estímulo que posibilita al animal el reconocimiento innato de una situación ambiental biológicamente significativa. A su vez, el movimiento instintivo, que es un movimiento de coordinación hereditaria y filogenéticamente pro- 
gramado, desatado por el estímulo desencadenante, controla esta situación con una capacidad innata (Lorenz, 1986, 144) $)^{5}$. Diversos investigadores han reconocido la necesidad de postular la existencia de un filtro para los estímulos. I.P. Paulov lo denominó detector, y hace más de medio siglo el ornitólogo norteamericano Francis Herrick afirmó que los instintos de una especie se ajustan entre sí como la llave a la cerradura. "Todavía hoy — dice Lorenzseguimos llamando estímulos-llave a las configuraciones de estímulos a que responde un MDI" (Lorenz, 1986, 147). Así, por ejemplo, la hembra del grillo ha desarrollado una estructura del órgano auditivo, que no es en absoluto capaz de oír otro sonido que el del canto de cortejo del macho (Lorenz, 1986, 146).

Así pues, los instintos son formas estables de movimiento o figuras de conducta innatas, especializadas y ordenadas a un objeto preciso, que son provocadas por excitadores altamente especializados y típicos, que cada espe-

5 Otras contribuciones a la noción de instinto proceden de eminentes etólogos y filósofos. N. Tinbergen (ganador del Nobel de fisiología y medicina de 1973, junto a von Frisch y Lorenz) por ejemplo, lo ha definido como "un comportamiento innato, es decir, un mecanismo nervioso, organizado jerárquicamente, sensible a determinados influjos ambientales que lo despiertan, lo ponen en funcionamiento y lo dirigen, a los que el instinto responde con movimientos bien coordinados que tienen por fin la conservación del individuo o de la especie" (Tinbergen, 1994, 166). W. Thorpe, por su parte, entiende que "el comportamiento innato o instinto es un mecanismo nervioso preformado, codificado genéticamente y organizado jerárquicamente para reaccionar ante determinados estímulos desencadenantes" (W. Thorpe, 1958, 17). 
cie animal encuentra en su mundo circundante, y hacia los que tal especie está genéticamente orientada (Gehlen, 1986, 125-126).

Frente a la conducta altamente especializada de los animales en general, excepto en el caso de los llamados animales curiosos, Lorenz define al hombre como el animal cuya especialización ha consistido en no especializarse, como el especialista de la inespecialización, tanto en lo relativo al sistema motriz, dotado en el hombre de una altísima plasticidad que no posee ningún otro animal, como en el sistema cognoscitivo-lingüístico-operativo (Lorenz, 1991, 252-253).

\section{Bibliografia empleada}

J. Benéitez, "Reflexiones sobre la naturaleza humana en el pensamiento de Aristóteles", Revista de filosofía, 2011, 36/1, 7-28.

A. Gehlen, Antbropologische Forschung, Hamburg, Rowohlt, 1961.

—, Urmensch und Spätkultur, Wiesbaden, Aula, 1986

J.H. Fabre, Souvenirs entomologiques, 10 vols., Paris, Ch. Delagrave, 1879-1907.

J.G. Herder, "Ensayo sobre el origen del lenguaje", J. G. Herder, Obra selecta, Madrid, Alfaguara, 1982.

—, „Ideen zur Philosophie der Geschichte der Menschheit“, J.G. Herder, Sämtliche Werke, Bd. 13, Berlin, Weidmannsche Buchhandlung, 1880; reprint Hildesheim, G. Olms Verlag, 1994.

I. Kant, „Idee zu einer allgemeinen Geschichte in weltbürgerlicher Absicht“, Kants Gesammelte Schriften, Bd. 8, Berlin-Boston, De Gruyter, 1969.

-, „Muthmaßlicher Anfang der Menschengeschichte“, Kants Gesammelte Schriften, Bd. 8, Berlin-Boston, De Gruyter, 1969. 
Historia de la idea antropobiológica

—, "Pedagogik (nach Rink)", Kants Gesammelte Schriften, Bd. 9, Berlin-Boston, De Gruyter, 1962.

K. Lorenz, Fundamentos de etología, Barcelona, Paidós, 1986.

—, L'altra faccia dello specchio, Milano, Adelphi, 1991.

F. Nietzsche, Zur Genealogie der Moral, zweite Auflage, Leipzig, C. G. Naumann, 1892.

A. Portmann, Biologie und Geist, Göttingen, Burgdorf Verlag, 1999, con introducción de Thure von Uexküll.

W. Thorpe, Learning and Instincts in Animal, London, Methuen and Co., 1958.

N. Tinbergen, Lo studio dell' istinto, Milano, Adelphi, 1994.

Tomás de Aquino, In III De Anima, Torino, Marietti, 1959.

—, Suma teológica, I, Madrid, BAC, 2001.

J. von Uexküll, Bedeuntugslehre, Leipzig, Verlag von J. A. Barth, 1940.

—, Streifzüge durch die Umwelten von Tieren und Menschen, Hamburg, Rowohlt, 1956.

Leopoldo José Prieto López

lprieto7@gmail.com 
